\title{
Systematic review and updated network meta-analysis comparing open, laparoscopic, and robotic pancreaticoduodenectomy
}

\author{
Alberto Aiolfi $^{1}$ (D) . Francesca Lombardo ${ }^{1} \cdot$ Gianluca Bonitta $^{1} \cdot$ Piergiorgio Danelli $^{2} \cdot$ Davide Bona $^{1}$
}

Received: 13 June 2020 / Accepted: 26 October 2020 / Published online: 14 December 2020

(c) The Author(s) 2020

\begin{abstract}
The treatment of periampullary and pancreatic head neoplasms is evolving. While minimally invasive Pancreaticoduodenectomy (PD) has gained worldwide interest, there has been a debate on its related outcomes. The purpose of this paper was to provide an updated evidence comparing short-term surgical and oncologic outcomes within Open Pancreaticoduodenectomy (OpenPD), Laparoscopic Pancreaticoduodenectomy (LapPD), and Robotic Pancreaticoduodenectomy (RobPD). MEDLINE, Web of Science, PubMed, Cochrane Central Library, and ClinicalTrials.gov were referred for systematic search. A Bayesian network meta-analysis was executed. Forty-one articles (56,440 patients) were included; 48,382 (85.7\%) underwent OpenPD, 5570 (9.8\%) LapPD, and 2488 (4.5\%) RobPD. Compared to OpenPD, LapPD and RobPD had similar postoperative mortality [Risk Ratio $(\mathrm{RR})=1.26$; 95\% CrI $0.91-1.61$ and $\mathrm{RR}=0.78 ; 95 \% \mathrm{CrI} 0.54-1.12)$ ], clinically relevant (grade $\mathrm{B} / \mathrm{C})$ postoperative pancreatic fistula $(\mathrm{POPF})(\mathrm{RR}=1.12$; 95\% CrI $0.82-1.43$ and $\mathrm{RR}=0.87$; 95\% CrI 0.64-1.14, respectively), and severe (Clavien-Dindo $\geq 3$ ) postoperative complications $(\mathrm{RR}=1.03$; 95\% CrI $0.80-1.46$ and $\mathrm{RR}=0.93$; 95\% CrI 0.65-1.14, respectively). Compared to OpenPD, both LapPD and RobPD had significantly reduced hospital length-of-stay, estimated blood loss, infectious, pulmonary, overall complications, postoperative bleeding, and hospital readmission. No differences were found in the number of retrieved lymph nodes and R0. OpenPD, LapPD, and RobPD seem to be comparable across clinically relevant POPF, severe complications, postoperative mortality, retrieved lymphnodes, and R0. LapPD and RobPD appears to be safer in terms of infectious, pulmonary, and overall complications with reduced hospital readmission We advocate surgeons to choose their preferred surgical approach according to their expertise, however, the adoption of minimally invasive techniques may possibly improve patients' outcomes.
\end{abstract}

Keywords Pancreaticoduodenectomy $\cdot$ Open pancreaticoduodenectomy $\cdot$ Laparoscopic pancreaticoduodenectomy $\cdot$ Robotic pancreaticoduodenectomy $\cdot$ Network meta-analysis

Electronic supplementary material The online version of this article (https://doi.org/10.1007/s13304-020-00916-1) contains supplementary material, which is available to authorized users.

Alberto Aiolfi

alberto.aiolfi86@gmail.com

Francesca Lombardo

francesca.lombardo89@gmail.com

Gianluca Bonitta

bbonit@icloud.com

Piergiorgio Danelli

piergiorgio.danelli@unimi.it

\section{Introduction}

Pancreaticoduodenectomy (PD) is a technically demanding surgical procedure that can provide cure or improved survival in patients with periampullary and pancreatic head diseases [1]. Indications for resection have increased because of the improvements in neoadjuvant treatment and surgical

Davide Bona

davide.bona@unimi.it

1 Department of Biomedical Science for Health, Division of General Surgery, Istituto Clinico Sant'Ambrogio, University of Milan, Via Luigi Giuseppe Faravelli, 16, 20149 Milan, Italy

2 Department of Biomedical and Clinical Sciences, "Luigi Sacco" Hospital, University of Milan, Milan, Italy 
technique $[2,3]$. There has been a growing interest towards minimally invasive techniques since the description of the first Laparoscopic Pancreaticoduodenectomy (LapPD) in 1994 [4]. Since then, LapPD has gained increasing acceptance with studies demonstrating its feasibility, safety and promising results [5]. Its reputation, however, was limited by the criticism regarding the protracted learning curve, potential for higher morbidity and the need for a high hospital volume to reach outcomes comparable to the open technique $[6,7]$. The growth of innovative robotic platforms has later opened new perspectives since the first Robotic-assisted Pancreaticoduodenectomy (RobPD) [8]. The better ergonomics, high-definition 3-D visualization, and increased motion allowed by the instruments with multiple degrees of freedom have contributed to the progressive worldwide spread of RobPD [9].

Previous studies have showed that LapPD and RobPD seem to be equally safe and were associated with reduced morbidity, shorter hospital length of stay, and non-inferior oncologic outcomes when compared to OpenPD [9-14]. The purpose of this network meta-analysis is to provide an updated evidence comparing short-term surgical and oncologic outcomes within pure OpenPD, LapPD, and RobPD.

\section{Materials and methods}

The systematic review was completed using the preferred reporting items for systematic reviews and network Metaanalyses guidelines (PRISMA-NMA) [15]. Approval from the local institutional review board was not necessary.

MEDLINE, Web of Science, PubMed, Cochrane Central Library and ClinicalTrials.gov were utilized for systematic search [16]. Articles published up to November 30, 2019 were screened. We searched for articles using the following search strategy: ("open pancreaticoduodenectomy" [tiab], OR "open pancreatoduodenectomy" [tiab]) AND ("laparoscopic pancreaticoduodenectomy" [tiab], OR "laparoscopic pancreatoduodenectomy" [tiab]); ("laparoscopic pancreaticoduodenectomy" [tiab], OR "laparoscopic pancreatoduodenectomy" [tiab]) AND ("robotic pancreaticoduodenectomy" [tiab], OR "robotic pancreatoduodenectomy" [tiab]); ("open pancreaticoduodenectomy" [tiab], OR "open pancreatoduodenectomy" [tiab]) AND ("robotic pancreaticoduodenectomy" [tiab], OR "robotic pancreatoduodenectomy" [tiab]). Titles and abstracts were assessed, inspected and references were screened. The PROSPERO study protocol registration number is CRD42020170952.

\section{Eligibility criteria}

Inclusion criteria includes (a) articles comparing surgical outcomes for either OpenPD, LapPD or RobPD in the setting of malignant, borderline or benign disease; (b) English-written studies; (c) articles with the longest follow-up or the largest sample size when two or more papers were published by the same institution, study group or used the same data-set; (d) studies released after the year 2003. Exclusion criteria are (a) non-English written articles; (b) studies without clear methodology and surgical technique; (c) articles reporting hybrid techniques (i.e., hand-assisted laparoscopic or robotic resections, combined laparoscopic-robotic approaches out of the standard); (d) studies with less than 20 patients per-arm comparison.

\section{Data extraction}

Retrieved records were authors, nation, year of publication, study design, patients' number, demographics, American Society of Anesthesiologists (ASA) score, surgical approach, postoperative surgical and oncologic outcomes. Three investigators (AA, FL, GB) individually extracted data from eligible articles and a fourth author (DB) clarified disagreements.

\section{Definitions}

PD was defined as any method of surgical removal of the pancreatic head, duodenum, and distal common bile duct. RobPD was defined as the use of robotic technique for PD, including resection and reconstruction without laparoscopic or hand-assisted techniques, nevertheless, this did include the use of laparoscopic ports by a surgical assistant which is considered as the standard practice. LapPD was defined as the complete use of a laparoscopic technique for PD, including resection and reconstruction without robotic or handassisted techniques.

Postoperative mortality was defined as either in-hospital or within 90-day mortality after PD. Postoperative Pancreatic Fistula (POPF) was classified in accordance with the International Study Group of Pancreatic Surgery (ISGPS); grade $\mathrm{B} / \mathrm{C}$ were considered clinically relevant [17]. Postoperative complications were recorded according to the Clavien-Dindo classification; grade $\geq 3$ were considered as severe postoperative complications [18]. Delayed Gastric Empting (DGE) was defined according to the ISGPS; grade $\mathrm{B} / \mathrm{C}$ were considered as clinically relevant [17].

\section{Quality evaluation}

The quality of observational studies was assessed with the Risk of Bias In Non-Randomized Studies (ROBINSI) instrument [19]. Confounding, selection, classification, intervention, missing data, outcomes measurement and reporting bias were considered. Each domain was estimated with "yes", "probably yes", "probably no" or "no" 
and studies were categorized as having low, moderate, serious, or critical risk of bias. The Cochrane risk of bias was adopted to appraise the quality of Randomized Controlled Trials (RCTs) and were graded as low risk (green circle), high risk (red circle), or unclear risk (yellow circle) of bias [20].

\section{Outcomes}

Primary outcomes were postoperative mortality, grade B/C POPF, severe postoperative complications (ClavienDindo $\geq 3$ ). Secondary outcomes were grade B/C DGE, Surgical Site Infection (SSI), pulmonary complications, bile leak, overall complications, estimated blood loss (ml), operative time (minutes), conversion to open, reoperation, hospital length of stay (HLOS) (days), postoperative bleeding, hospital readmission, R0, harvested lymphnodes $(n)$, and costs (in \$).

\section{Statistical analysis}

A systematic review and a comprehensive Bayesian network analysis were executed [21-23]. Risk Ratio (RR) and estimated mean difference (md) were used as pooled effect size measure for binary and continuous outcomes. For the between-study variability $(\tau)$ we used an informative halfnormal prior with zero mean and scale 0.5 [24]. To assess local inconsistencies, the node-splitting method and prior distribution sensitivity analysis were measured $[25,26]$. Heterogeneity $\left(I^{2}\right)$ was defined as low $(<25 \%)$, moderate $(25-75 \%)$, or high $(>75 \%)$ [27]. The inference was performed using mean and 95\% Credible Intervals (CrI) and was considered significant when it encompasses the null hypothesis value. The transitivity assumption (i.e., studies comparing different sets of interventions needed to be sufficiently similar) was considered to provide valid indirect inferences. To assess transitivity, we generated descriptive statistics and compare the distributions of baseline characteristics across studies and treatment comparisons. The accuracy of the inference was assessed by convergence of MCMC algorithm [28]. The treatment ranking probability was estimated with the cumulative ranking curve (SUCRA). The network geometry was appraised and the confidence of outcomes estimates was assessed with Confidence in Network Meta-Analysis (CINeMA) instrument. Jags and R-Cran were used for statistical analyses [29].

\section{Results}

\section{Systematic review}

Five thousand nine hundred and twenty-three titles were found using the described criteria. After removing duplicates, 4022 publications were revised with 41 studies fulfilling the inclusion criteria (Fig. 1). Of the included studies, three were RCTs. None of the studies received a low risk of bias on all assessed items. Because the lack of patients and/ or outcomes assessors blinding, all trials were graded as having high/unclear risk of performance and detection bias. Because the individual surgeon experience was not precisely indicated, other bias was defined as high (Supplementary Fig. 1). Thirty-eight articles were observational non-randomized studies. According to the ROBINS-I tool, 21 studies were categorized as having moderate risk of bias while 17 were categorized as having severe risk of bias. Outcomes might have been influenced by confounding and selection bias because inclusion/exclusion criteria and patient treatment allocation were heterogeneous among studies (Supplementary Table 1). The assessments of confidence in the estimates using CINeMA showed low to very low confidence, essentially due to study limitation, imprecision, and heterogeneity.

Patient demographics and preoperative characteristics are summarized in Table 1. Overall, 56,440 patients were included in the quantitative analysis, out of which 48,382 (85.7\%) underwent OpenPD, 5570 (9.8\%) LapPD, and 2488 $(4.5 \%)$ RobPD. The median age (range) was 61.5 years (18-89). Gender was specified in 45,521 patients: 23,054 patients $(50.6 \%)$ were male and 22,467 (49.4\%) were females. BMI was defined in 26,253 patients; the median (range) BMI was 25.2 (15-49.6). ASA score was reported in 20,812 patients (20 studies) with $10,408(50.1 \%)$ being classified as having ASA $\geq 3$. Overall, $92.5 \%$ of patients underwent surgery for malignancy, $55.9 \%$ for pancreatic ductal adenocarcinoma, and $7.5 \%$ for borderline or benign tumor. Tumor size was defined in 21 studies and ranged from 0.1 to $14 \mathrm{~cm}$. Neoadjuvant therapy was reported in 25 studies and consisted in any preoperative chemotherapy, radiation therapy or both. Cost analysis was reported in six studies.

\section{Meta-analysis}

\section{Postoperative mortality}

Thirty-eight studies (54,179 patients) reported this outcome (Fig. 2a). No significant differences were found comparing LapPD vs. OpenPD ( $R R=1.26$; 95\% CrI 0.91-1.61) and RobPD vs. OpenPD ( $\mathrm{RR}=0.78 ; 95 \% \mathrm{CrI} 0.54-1.12)$. The global heterogeneity was low $\left(I^{2}=18.2 \%\right.$; $95 \% \mathrm{CrI}$ 

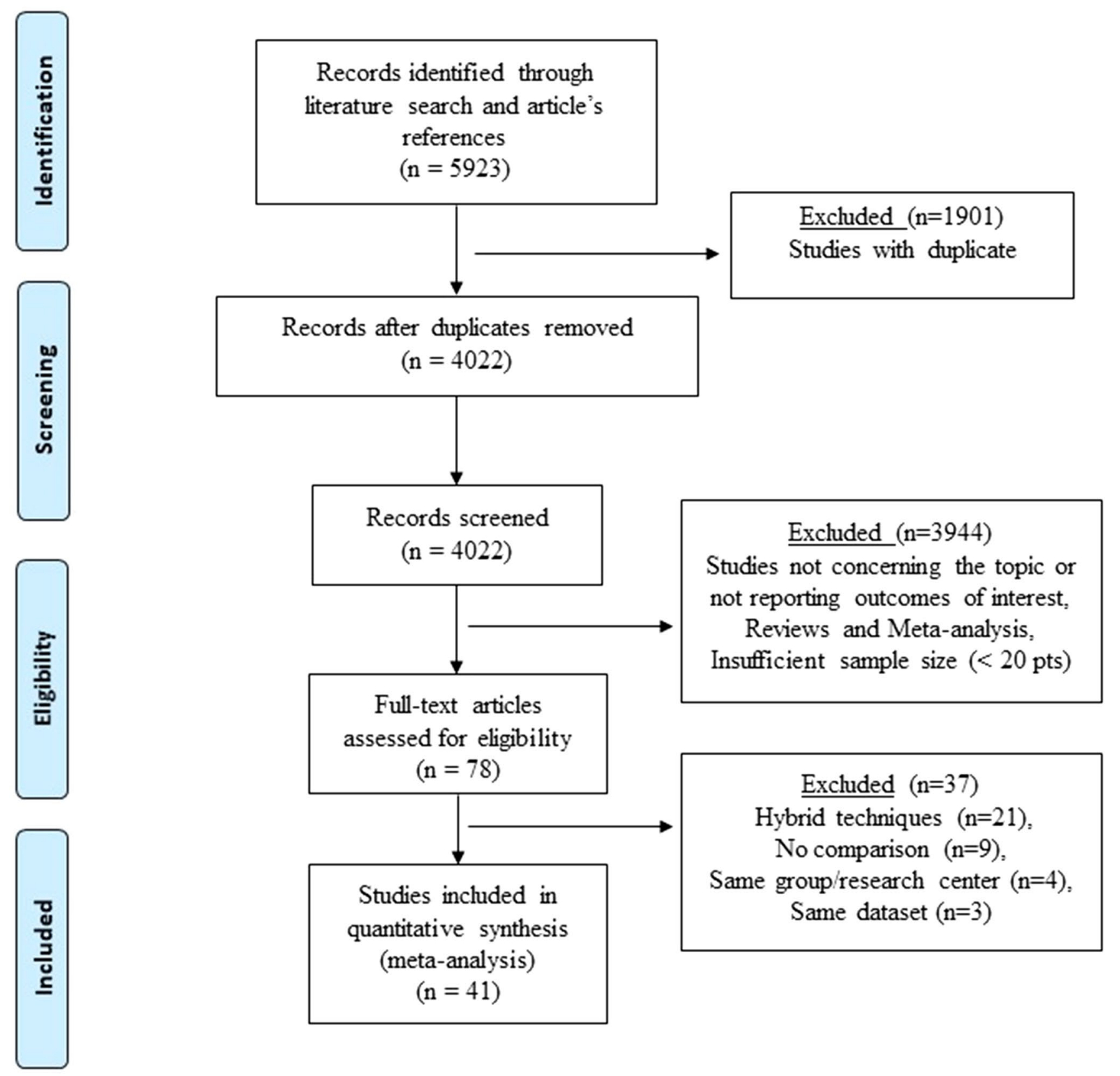

Fig. 1 The Preferred Reporting Items for Systematic Reviews and network meta-analyses checklist (PRISMA-NMA) diagram

10.1-29.2\%). The SUCRA ranking was $94 \%$ for LapPD, $52 \%$ for OpenPD, and 4\% for RobPD (Supplementary Fig 2A). The node splitting analysis does not show evidence of local inconsistency and the sensitivity analysis yields closer results for LapPD vs. OpenPD $(\mathrm{RR}=1.31 ; 95 \% \mathrm{CrI}$ 0.95-2.03) and RobPD vs. OpenPD $(\mathrm{RR}=0.72 ; 95 \% \mathrm{CrI}$ $0.45-1.13)$.

\section{Grade B/C POPF}

Thirty-six studies $(50,974$ patients) reported this outcome (Fig. 2b). No significant differences were noticed for LapPD vs. OpenPD ( $R R=1.12 ; 95 \% \mathrm{CrI} 0.82-1.43)$ and RobPD vs. OpenPD $(\mathrm{RR}=0.87 ; 95 \% \mathrm{CrI} 0.64-1.14)$. The global heterogeneity was low $\left(I^{2}=21.2 \%\right.$; 95\% CrI $\left.13.4-28.6 \%\right)$. The SUCRA ranking was $78 \%$ for LapPD, $58 \%$ for OpenPD, and $13 \%$ for RobPD (Supplementary Fig. 2B). The node splitting analysis does not show evidence of local inconsistency and the sensitivity analysis produces closer results for LapPD 


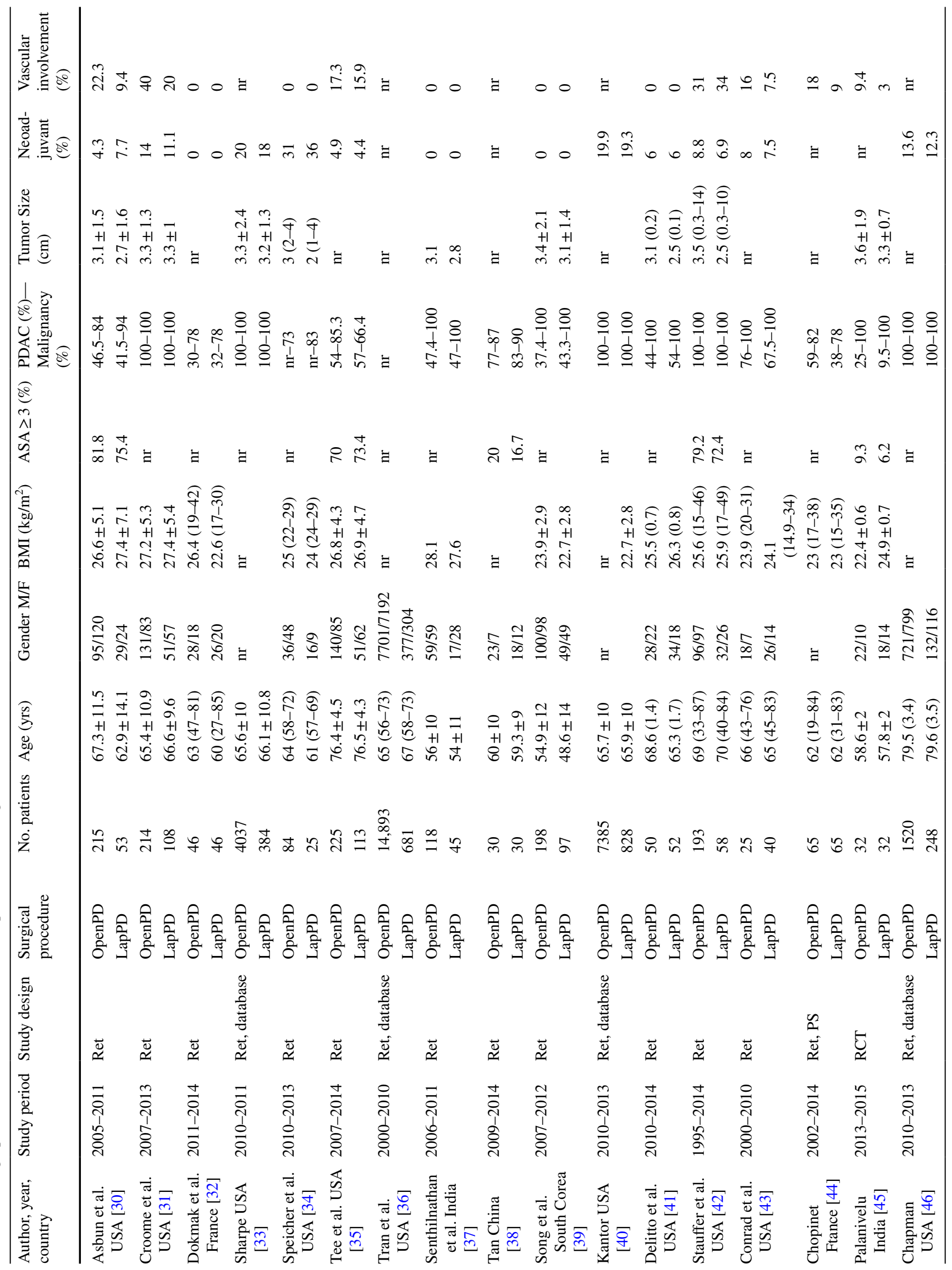




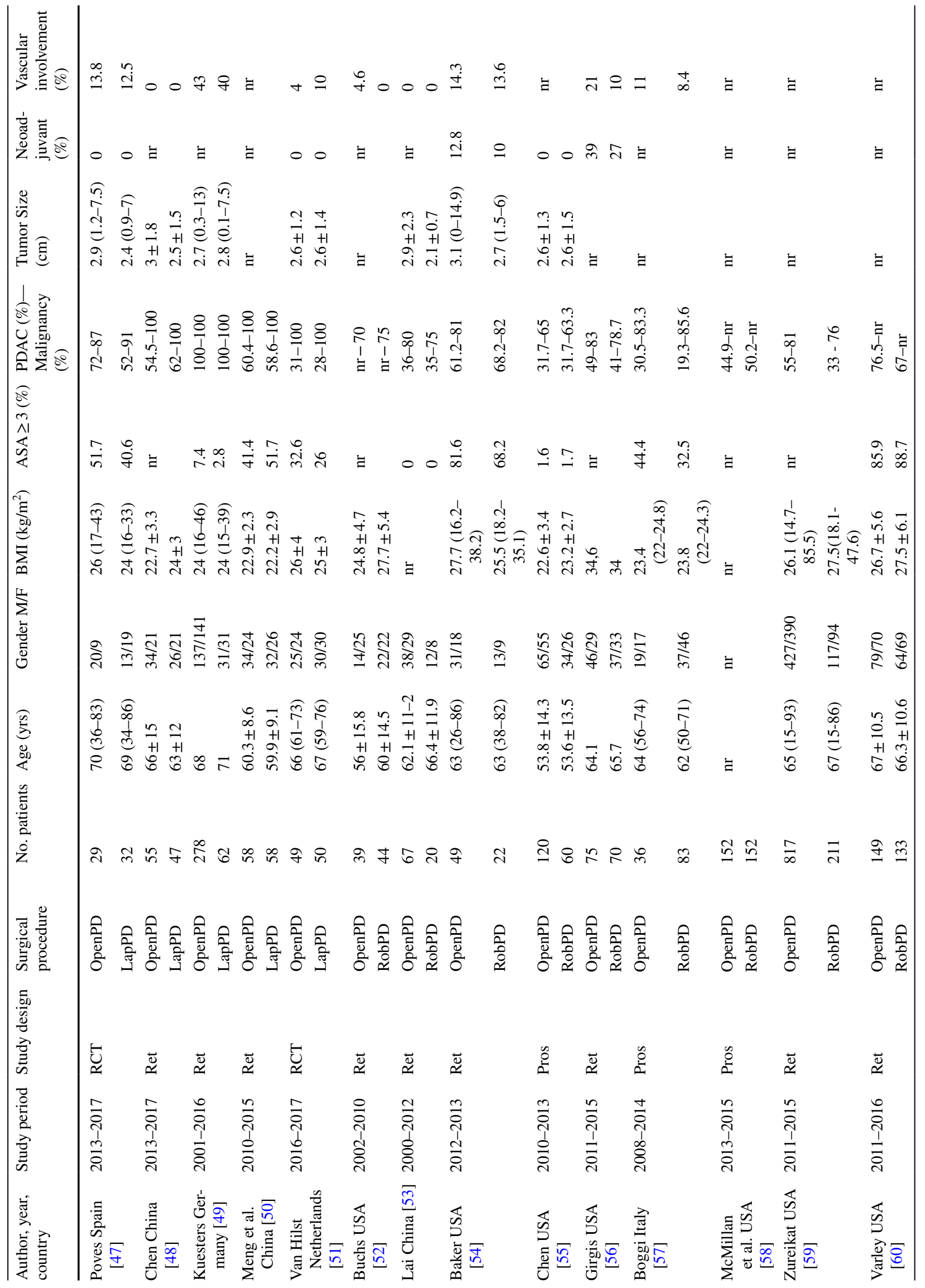




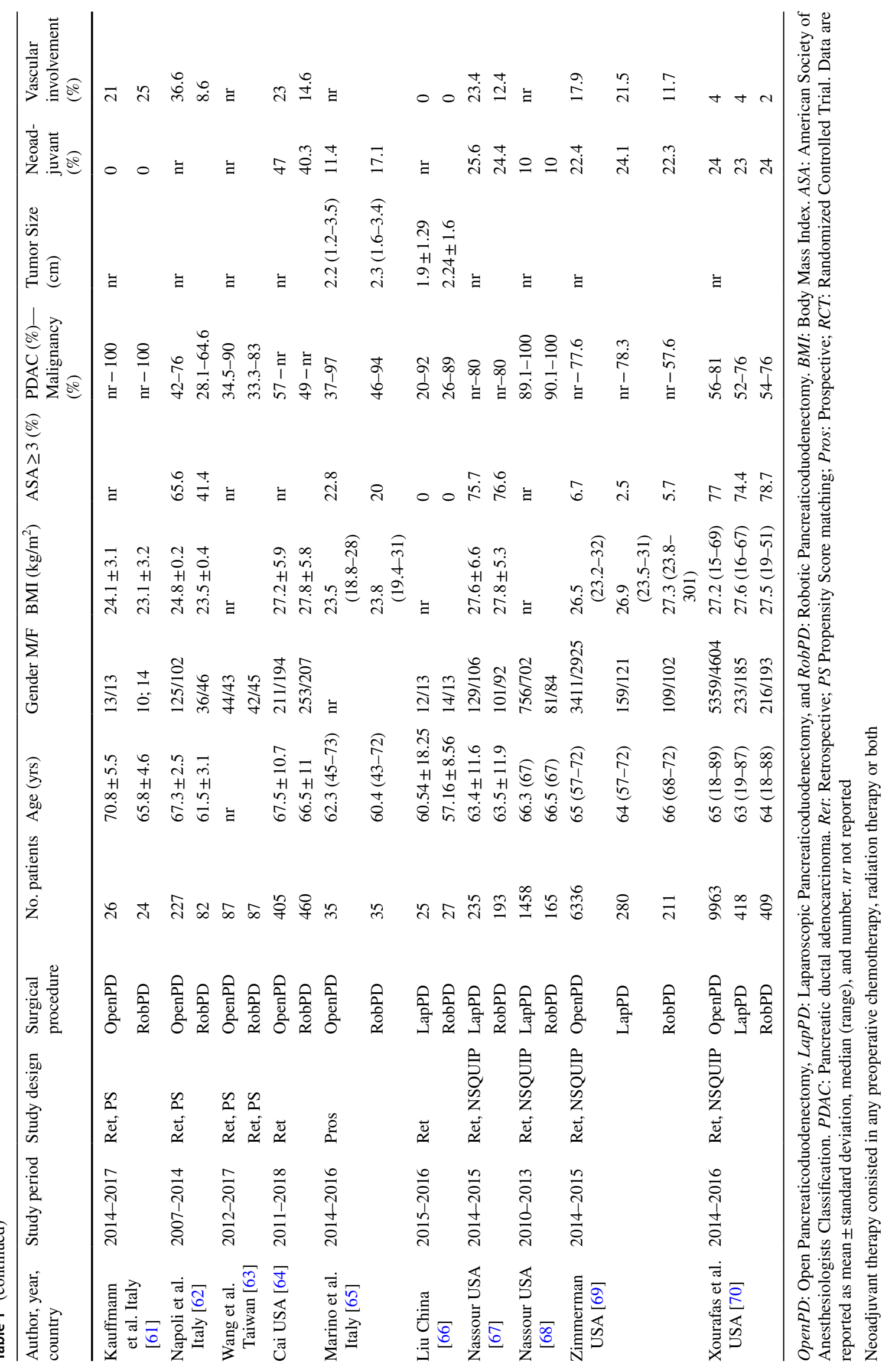


vs. OpenPD ( $R R=1.14 ; 95 \% \mathrm{CrI} 0.84-1.47)$ and RobPD vs. OpenPD $(\mathrm{RR}=0.85 ; 95 \% \mathrm{CrI} 0.61-1.13)$.

\section{Severe postoperative complication (Clavien-Dindo $\geq 3$ )}

Twenty-eight studies (48,921 patients) reported this outcome (Fig. 2c). No significant differences were found comparing LapPD vs. OpenPD ( $R R=1.03 ; 95 \% \mathrm{CrI} 0.80-1.46)$ and RobPD vs. OpenPD (RR $=0.93 ; 95 \% \mathrm{CrI} 0.65-1.14)$. The global heterogeneity was low $\left(I^{2}=24.2 \%\right.$; $95 \% \mathrm{CrI}$ 15.6-31.8\%). The SUCRA ranking was $68 \%$ for LapPD, $53 \%$ for OpenPD, and 29\% for RobPD (Supplementary Fig. 2C). The node splitting analysis does not show evidence of local inconsistency and the sensitivity analysis shows similar results for LapPD vs. OpenPD $(R R=1.15$; 95\% CrI 0.89-1.52) and RobPD vs. OpenPD (RR $=0.86$; 95\% CrI 0.71-1.29).

\section{Secondary outcomes}

The pooled analysis shows a significantly reduced RR comparing LapPD vs. OpenPD and RobPD vs. OpenPD in terms of surgical site infection (20 studies) $(\mathrm{RR}=0.71 ; 95 \% \mathrm{CrI}$ $0.59-0.83$ and $\mathrm{RR}=0.68 ; 95 \% \mathrm{CrI} 0.54-0.81$, respectively), pulmonary complications (18 studies) $(\mathrm{RR}=0.81 ; 95 \% \mathrm{CrI}$ $0.70-0.94$ and $\mathrm{RR}=0.73 ; 95 \% \mathrm{CrI} 0.64-0.86$, respectively), overall complications ( 25 studies) $(\mathrm{RR}=0.81 ; 95 \% \mathrm{CrI}$ $0.74-0.93$ and $\mathrm{RR}=0.79 ; 95 \% \mathrm{CrI} 0.72-0.91$, respectively), and hospital readmission (17 studies) $(\mathrm{RR}=0.81 ; 95 \% \mathrm{CrI}$ 0.70-0.94 and $\mathrm{RR}=0.73 ; 95 \% \mathrm{CrI} 0.64-0.86$, respectively).

Compared to OpenPD, both LapPD and RobPD showed a significantly reduced HLOS (35 studies) (md -1.9 ; $95 \% \mathrm{CrI}-3.01,-0.77$ and $-2.23 ; 95 \% \mathrm{CrI}-3.6,-0.99$, respectively), estimated intraoperative blood loss (29 studies) ( $\mathrm{md}-148.5$; 95\% CrI - 156.5, -140.5 and -158.5 ; $95 \% \mathrm{CrI}-169.2,-147.9$, respectively), and postoperative bleeding ( 23 studies) $(\mathrm{RR}=0.64 ; 95 \% \mathrm{CrI} 0.48-0.81$ and $\mathrm{RR}=0.67 ; 95 \% \mathrm{CrI} 0.42-0.82$, respectively). Operative time was significantly longer when comparing LapPD vs. OpenPD and RobPD vs. OpenPD (md 60.9; 95\% CrI 53.9-67.98 and $\mathrm{md}=33.1$; 95\% CrI 24.02-42.3, respectively) (32 studies). RobPD was associated with significantly reduced risk of conversion to open compared to LapPD $\left(\mathrm{RR}=0.71 ; 95 \%\right.$ CrI $\left.0.59-0.83 ; I^{2}=15.1 \%\right)$. No significant differences were found comparing grade B/C DGE (25 studies), bile leak (14 studies), reoperation (26 studies), R0 (27 studies), and harvested lymph nodes (28 studies) across the three surgical approaches. The league table for all measured outcomes was shown in Table 2. SUCRA ranking is reported in Supplementary Fig. 2.

\section{Discussion}

This study showed that pure OpenPD, LapPD, and RobPD appears to be equally safe with comparable postoperative mortality, grade B/C POPF, and severe postoperative complications (Clavien-Dindo $\geq 3$ ). Compared to OpenPD, LapPD and RobPD seems to be associated with significantly reduced blood loss, hospital length of stay, readmission, infectious, pulmonary, and overall complications. R0 margins and total number of harvested lymph nodes were similar across treatments.

Over the last two decades, substantial improvements have been demonstrated in the management of pancreatic head and periampullary neoplasms with an increased enthusiasm for minimally invasive approaches [71, 72]. LapPD has been shown to be comparable to Open PD in terms of safety and oncologic results [2]. However, technical limitation and poor ergonomics have made radical oncological dissection and anastomosis fashioning challenging. The advent of robotic platforms has brought new enthusiasm because of the better ergonomics and high-definition 3D visualization. Furthermore, its improved instruments motion range has led to an enhanced dissection of the uncinate process, retroportal lamina propria, and lymphnodes combined with an easier anastomosis fashioning [9]. Despite these technical advancements, postoperative mortality and morbidity are reported up to 3\% and 30-40\% in referral centers [3]. This network analysis showed that postoperative mortality was equivalent comparing OpenPD, LapPD, and RobPD with a low related heterogeneity $\left(I^{2}=18.3 \%\right)$, thus demonstrating a minor divergence within studies. Notably, the recent advancements of critical care have significantly improved postoperative course with reduced mortality therefore, this effect should be considered while interpreting this result. Additionally, preoperative patient selection bias, surgeon learning curve, hospital volume, and the non-specified individual-patient cause of death may be potential source of bias. Clinically relevant POPF (Grade B/C) has been shown to be a foremost contributor to major morbidity, mortality and has been reported in up to $20 \%$ of patients [73]. Independent risk factors for POPF include age, technique for anastomosis, patient comorbidities, size and consistency of the pancreatic duct, parenchyma, and low cardiopulmonary reserve [74]. We found that grade B/C POPF was comparable among the OpenPD, LapPD, and RobPD groups. This result should be interpreted cautiously because of variability in surgical techniques, postoperative prophylactic octreotide use, pancreatic duct diameter, gland texture (soft vs. firm/ hard), surgical anastomotic techniques (invagination vs. duct-to-mucosa), surgeons' experience/learning curve, and hospital volume. However, the related heterogeneity was low $\left(I^{2}=21.2 \%\right)$ with narrow $95 \% \mathrm{CrI}$, thus adding further 

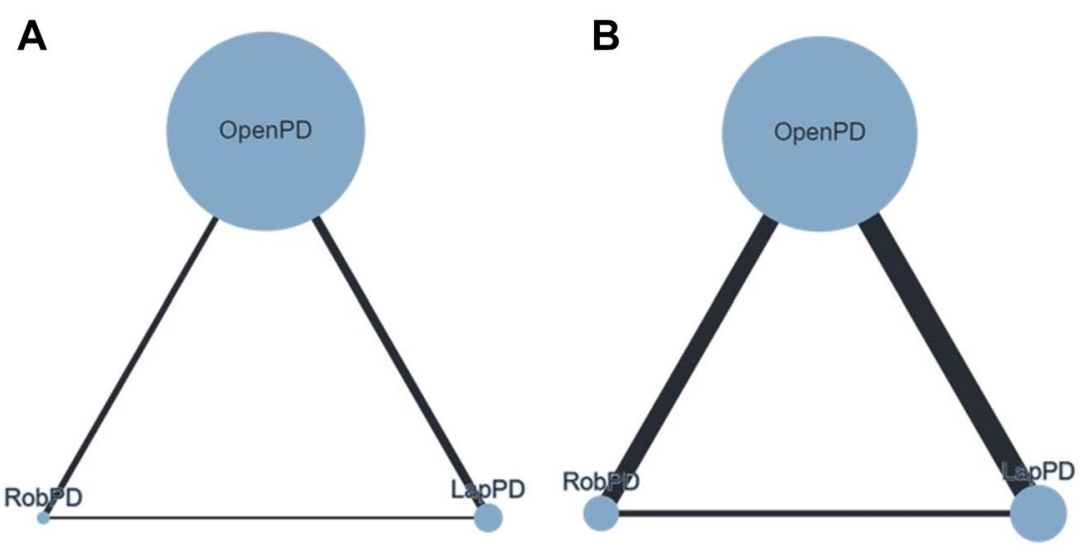

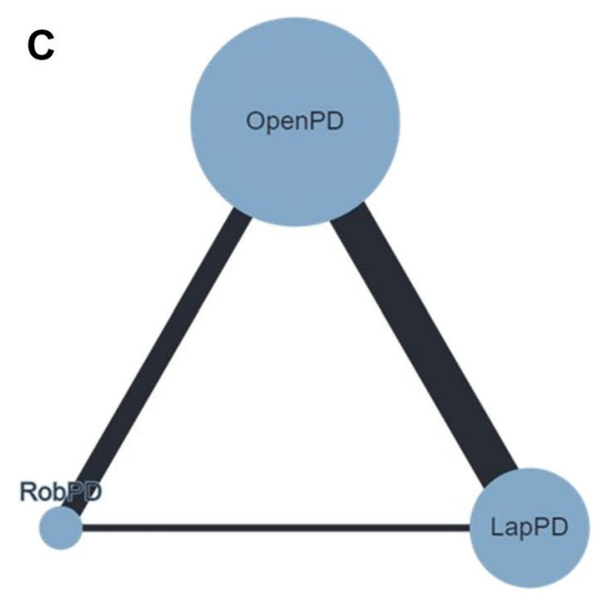

Fig. 2 Network geometry for studies reporting: (a) Postoperative mortality, (b) grade B/C POPF, (c) Clavien-Dindo 3/4. The nodes reflect the surgical approaches (OpenPD, LapPD, and RobPD) while

consistency to the result. Severe postoperative complications (Clavien-Dindo $\geq 3$ ) were found to be equivalent comparing OpenPD, LapPD and RobPD. The related global heterogeneity was low $\left(I^{2}=24.2 \%\right)$. However, baseline comorbidities and the heterogeneity in studies reporting may marginally influence this variability. Despite the lack of statistical significance, the SUCRA evaluation ranked RobPD as the surgical approach with the lowest probability to be ranked as first treatment for mortality (4\%), POPF (13\%), and postoperative Clavien-Dindo $\geq 3$ (29\%).

Compared to OpenPD, LapPD and RobPD were associated with a significantly reduced infectious, pulmonary, and overall complications. This is probably due to the reduced tissue trauma, postoperative pain, pulmonary impairment, and systemic stress response [2, 12]. Similarly, LapPD and RobPD were associated with significantly reduced blood loss, hospital length of stay, and hospital readmission when compared to OpenPD. The decreased blood loss and reduced transfusion requirement may presumably preserve patients' immune system with a possibility of enhancing anti-neoplasm response [8, 12]. This is in line with the article by Kazanjian and colleagues, that reported improved survival rate following PD for pancreatic head neoplasm in patients with limited operative blood loss $(<400 \mathrm{ml})$ [75]. Moreover, the reduced SSI, pulmonary, and overall complications may allow an easier access to adjuvant treatments and potentially improve survival [76]. However, results should be interpreted cautiously because of the moderate/ high related heterogeneity, probably influenced by patients' comorbidities, preoperative patients' selection, BMI, antibiotic therapy, ASA grade, smoke status, tumor types and size, surgical technique, need for vascular resection, and surgeon experience. the connecting edge reflect the treatment comparison. Nodes size reflects the sample size while edges width reflects the number of studies for a specific pairwise comparison

Tumor free resection margin (R0) and total number of harvested lymph nodes were similar across treatments. Again, these results need to be cautiously interpreted because of possible confounders related to different tumor size, histology, grading, presence of perineural infiltration, vascular resection, and neoadjuvant treatment. Compared to LapPD, RobPD was associated with a significantly reduced conversion rate (RR $0.71 ; 95 \% \mathrm{CrI} 0.60-0.89 ; I^{2}=15.1 \% \%$ ). This may be attributable to a more precise dissection in narrow spaces, better ergonomics, improved stability, articulated instruments manoeuvrability with highly defined 3-D anatomical dissection planes and neurovascular structures visualization $[11,12]$.

Opponents to minimally invasive technique may argue that longer operative times, overall increased health-care costs combined with the limited superiority do not justify the use of minimally invasive techniques. This network analysis showed statistically significant longer operative time for LapPD and RobPD, might be due to the learning curve and docking of the robot. Few articles reported the analysis of costs with a trend towards greater cost in minimally invasive techniques, mainly RobPD, probably due to the maintenance of instrument and equipment costs [77]. However, the reduced postoperative complications, hospital length of stay, and hospital readmissions may suggest a presumed ultimate cost-effectiveness [78].

LapPD and RobPD are associated with procedure-specific learning curves, however, conclusive results assessing the number of procedures required to overcome the learning curve are still inconclusive. Speicher et al. in their single high-volume center study demonstrated a significant reduction in operating time after 10 LapPD and significant reduction of blood loss after 50 procedures compared to OpenPD [34]. Sharpe et al. showed in their national database study, a 
Table 2 League table

\begin{tabular}{|c|c|c|c|}
\hline LapPD & $0.81(0.65-1.16)$ & $0.66(0.45-1.08)$ & Postoperative mortality \\
\hline $1.26(0.91-1.61)$ & OpenPD & $0.78(0.54-1.12)$ & \\
\hline $1.49(0.89-2.03)$ & $1.23(0.90-1.84)$ & RobPD & \\
\hline LapPD & $0.95(0.74-1.22)$ & $0.82(0.58-1.13)$ & Grade B/C POPF \\
\hline $1.12(0.82-1.43)$ & OpenPD & $0.87(0.64-1.14)$ & \\
\hline $1.21(0.88-1.71)$ & $1.15(0.87-1.55)$ & RobPD & \\
\hline LapPD & $0.96(0.72-1.26)$ & $0.89(0.59-1.29)$ & Clavien-Dindo $\geq 3$ \\
\hline $1.03(0.80-1.46)$ & OpenPD & $0.93(0.65-1.14)$ & \\
\hline $1.12(0.78-1.69)$ & $1.07(0.75-1.57)$ & RobPD & \\
\hline LapPD & $1.05(0.72-1.38)$ & $0.93(0.69-1.57)$ & Grade B/C DGE \\
\hline $0.98(0-83-1.23)$ & OpenPD & $0.89(0.72-1.16)$ & \\
\hline $1.08(0.84-1.76)$ & $1.12(0.81-1.47)$ & RobPD & \\
\hline LapPD & $1.34(1.11-2.05)$ & $0.91(0.68-1.35)$ & Surgical Site Infection \\
\hline $0.71(0.59-0.83)$ & OpenPD & $0.68(0.54-0.81)$ & \\
\hline $1.08(0.76-1.54)$ & $1.41(1.07-1.98)$ & RobPD & \\
\hline LapPD & $1.18(1.09-1.75)$ & $0.94(0.70-1.23)$ & Pulmonary Complications \\
\hline $0.81(0.70-0.94)$ & OpenPD & $0.73(0.64-0.86)$ & \\
\hline $1.05(0.81-1.40)$ & $1.29(1.07-2.41)$ & RobPD & \\
\hline LapPD & $0.94(0.76-1.57)$ & $0.94(0.66-1.34)$ & Bile Leak \\
\hline $1.07(0.76-1.61)$ & OpenPD & $0.98(0.35-1.29)$ & \\
\hline $1.09(0.78-1-33)$ & $1.05(0.79-1.51)$ & RobPD & \\
\hline LapPD & $1.23(1.16-2.34)$ & $0.97(0.86-1.19)$ & Overall Complications \\
\hline $0.81(0.74-0.93)$ & OpenPD & $0.79(0.72-0.91)$ & \\
\hline $1.03(0.94-1.13)$ & $1.34(1.12-2.06)$ & RobPD & \\
\hline LapPD & $1.08(0.86-1.43)$ & $0.89(0.61-1.07)$ & Reoperation \\
\hline $0.94(0.69-1.25)$ & OpenPD & $0.71(0.49-1.09)$ & \\
\hline $1.12(0.95-1.75)$ & $1.22(0.91-1.52)$ & RobPD & \\
\hline LapPD & $1.18(1.07-1.89)$ & $0.94(0.65-1.71)$ & Hospital readmission \\
\hline $0.81(0.70-0.94)$ & OpenPD & $0.73(0.64-0.86)$ & \\
\hline $1.05(0.78-1.72)$ & $1.12(1.03-1.91)$ & RobPD & \\
\hline LapPD & $1.21(0.91-1.53)$ & $0.87(0.53-1.29)$ & R0 \\
\hline $0.83(0.68-1.59)$ & OpenPD & $1.13(0.82-1.66)$ & \\
\hline $1.12(0.86-1.30)$ & $0.85(0.67-1.36)$ & RobPD & \\
\hline LapPD & $1.06(0.82-1.33)$ & $0.54(0.38-0.74)$ & Vascular resection \\
\hline $0.94(0.75-1.21)$ & OpenPD & $0.51(0.37-0.69)$ & \\
\hline $1.84(1.34-2.62)$ & $1.95(1.47-2.63)$ & RobPD & \\
\hline LapPD & $148.5(140.5-156.5)$ & $-10.04(-23.3,3.21)$ & Estimated Blood Loss \\
\hline$-148.5(-156.5-140.5)$ & OpenPD & $-158.5(-169.2-147.9)$ & \\
\hline $10.04(-3.21,23.3)$ & $158.5(147.9-169.2)$ & RobPD & \\
\hline LapPD & $-1.68(-3.63,0.26)$ & $0.51(-3.43,4.45)$ & Harvested Lymphnodes \\
\hline $1.68(-0.26,3.64)$ & OpenPD & $2.18(-1.23,5.61)$ & \\
\hline$-0.51(-4.45,3.42)$ & $-2.18(-5.61,1.23)$ & RobPD & \\
\hline LapPD & $1.92(0.77-3.01)$ & $-0.30(-1.93,1.2)$ & HLOS \\
\hline$-1.9(-3.01,-0.77)$ & OpenPD & $-2.23(-3.6,-0.99)$ & \\
\hline $0.3(-1.2,1.93)$ & $2.23(0.99-3.6)$ & RobPD & \\
\hline LapPD & $-60.9(-67.98,-53.9)$ & $-27.8(-38.6,-17.1)$ & Operative time \\
\hline $60.9(53.9-67.98)$ & OpenPD & $33.1(24.02-42.3)$ & \\
\hline $27.8(17.1-38.6)$ & $-33.1(-42.3,-24.0)$ & RobPD & \\
\hline
\end{tabular}

OpenPD Open Pancreaticoduodenectomy, LapPD Laparoscopic Pancreaticoduodenectomy, RobPD (obotic Pancreaticoduodenectomy. POPF: Postoperative pancreatic fistula; DGE: Delayed gastric emptying; HLOS: Hospital length of stay

Values are expressed as Risk Ratio $(R R)$ and 95\% Credible Intervals $(95 \% \mathrm{CrI})$

Values in each column represent the relative effect of the referral treatment (bold) with the comparator 
comparable LapPD-related postoperative mortality after 10 operations with OpenPD [33]. Choi et al. concluded that 40 LapPD is the minimum number of procedures necessary to reach technical competence [79]. In the setting of RobPD, Boone et al. showed in their single high-volume center study, several inflection points associated with significant improvement in estimated blood loss and conversion rate (20 cases), reduction of POPF (40 cases), and improvement in operative time (80 cases) [80]. Pancreatic resection in high-volume teaching centres with adequate preoperative training programs, intraoperative coaching, and dedicated staff has been reported to be associated with significant improvements in surgical outcomes, quality of surgical resection, and survival [81]. Therefore, while the majority of included studies were performed in high-volume center, our results may not be generalized to small community hospitals.

Limitations related to exclusion of non-English written articles and heterogeneity for some of the included studies (i.e., patients' demographics, comorbidities, inclusion/ exclusion criteria, variability in operative technique, and pathological data) should be considered. Detailed cancer staging, histologic subtype, neoadjuvant treatment, type of induction therapy, and tumor location are lacking in some studies and reported as aggregated in other studies. Given that these clinical factors may impact outcomes, the lack of these components should be considered as additional confounder. In addition, patients' treatment allocation was heterogeneous among studies and may denote a preoperative confounding and selection bias. Only three RCTs were included in the final analysis with most of the studies being low-quality non-randomized observational studies. This constitute the principal limitation of this meta-analysis and should be considered while interpreting our results. Imprecision must be considered in some of the outcomes because of the credible interval crossing the null value or include values favouring either treatment. The treatment ranking should be cautiously interpreted because it does not consider the magnitude of differences in effects between treatments and therefore chance may explain any apparent difference. Finally, no data were available on postoperative medium-/ long-term survival effect of minimally invasive approaches and this mandates further investigations.

We believe that this network meta-analysis updates and broadens Ricci et al. and Kamarajah and colleagues' studies $[11,12]$. The research focus on pure surgical approaches. Therefore, articles which include hybrid hand-assisted and differently combined demolitive/reconstructive laparoscopic-robotic technique, out of the standard approaches were excluded in attempt to obtain more homogeneous data. Second, in effort to control the effect of the early learning curve and obtain more solid data, studies with less than 20 patients per arm were excluded a priori. The study was intended in accordance with PRISMA guidelines and followed a robust methodology apriori registered in the PROSPERO protocol. This generates a homogenous cohort of patients as confirmed by low heterogeneity seen in the primary outcomes.

\section{Conclusions}

This network meta-analysis shows that the treatment of periampullary and pancreatic head tumors is evolving. Pure OpenPD, LapPD, and RobPD seems to be equivalent in terms of safety. Compared to OpenPD, both LapPD and RobPD seem associated with reduced risk of infectious, pulmonary, overall complications, blood loss, postoperative bleeding, hospital length of stay, and hospital readmission. Retrieved lymph nodes, tumor-free resection margins, clinically relevant POPF, severe postoperative complications, and clinically relevant DGE appear to be comparable. We advocate surgeons to choose their preferred surgical approach according to their expertise however, the adoption of minimally invasive techniques may possibly improve patients' outcomes.

\section{Acknowledgements None.}

Funding Open access funding provided by Università degli Studi di Milano within the CRUI-CARE Agreement.

Data availability Data generated at a central, large-scale facility, available upon request.

\section{Compliance with ethical standards}

Conflict of interest The authors declare that they have no conflict of interest.

Ethical approval For this type of article ethical approval is not required because does not contain any studies with human participants or animals performed by any of the authors.

Human and animal rights This study does not contain any studies with human participants or animals performed by any of the authors.

Informed consent For this type of study, formal consent was not necessary.

Open Access This article is licensed under a Creative Commons Attribution 4.0 International License, which permits use, sharing, adaptation, distribution and reproduction in any medium or format, as long as you give appropriate credit to the original author(s) and the source, provide a link to the Creative Commons licence, and indicate if changes were made. The images or other third party material in this article are included in the article's Creative Commons licence, unless indicated otherwise in a credit line to the material. If material is not included in the article's Creative Commons licence and your intended use is not permitted by statutory regulation or exceeds the permitted use, you will need to obtain permission directly from the copyright holder. To view a copy of this licence, visit http://creativecommons.org/licenses/by/4.0/. 


\section{References}

1. Tempero MA, Malafa MP, Al-Hawary M et al (2017) Pancreatic Adenocarcinoma, NCCN clinical practice guidelines in oncology. J Natl Compr Canc Netw 15(8):1028-1061

2. Nickel F, Haney CM, Kowalewski KF et al (2020) Laparoscopic versus open pancreaticoduodenectomy: a systematic review and meta-analysis of randomized controlled trials. Ann Surg 271(1):54-66

3. Hackert T, Probst P, Knebel P et al (2018) Pylorus resection does not reduce delayed gastric emptying after partial pancreatoduodenectomy: a blinded randomized controlled trial (PROPP Study, DRKS00004191). Ann Surg 267:1021-1027

4. Gagner M, Pomp A (1994) Laparoscopic pylorus-preserving pancreatoduodenectomy. Surg Endosc 8(5):408-410

5. van Hilst J, de Rooij T, Abu Hilal M et al (2017) Worldwide survey on opinions and use of minimally invasive pancreatic resection. HPB (Oxford) 19(3):190-204

6. Nappo G, Perinel J, Bechwaty El et al (2016) Minimally invasive pancreatic resection: is it really the future? Dig Surg 33(4):284-289

7. Adam MA, Thomas S, Youngwirth L et al (2017) Defining a hospital volume threshold for minimally invasive pancreaticoduodenectomy in the United States. JAMA Surg 152:336-342

8. Giulianotti PC, Coratti A, Angelini M et al (2003) Robotics in general surgery: personal experience in a large community hospital. Arch Surg 138(7):777-784

9. Podda M, Gerardi C, Di Saverio S et al (2020) Roboticassisted versus open pancreaticoduodenectomy for patients with benign and malignant periampullary disease: a systematic review and meta-analysis of short-term outcomes. Surg Endosc 34(6):2390-2409

10. Jiang YL, Zhang RC, Zhou YC (2019) Comparison of overall survival and perioperative outcomes of laparoscopic pancreaticoduodenectomy and open pancreaticoduodenectomy for pancreatic ductal adenocarcinoma: a systematic review and meta-analysis. BMC Cancer 19(1):781

11. Kamarajah SK, Bundred J, Marc OS et al (2020a) Robotic versus conventional laparoscopic pancreaticoduodenectomy a systematic review and meta-analysis. Eur J Surg Oncol 46(1):6-14

12. Ricci C, Casadei R, Taffurelli G et al (2018) Minimally invasive pancreaticoduodenectomy: what is the best "choice"? A systematic review and network meta-analysis of non-randomized comparative studies. World J Surg 42(3):788-805

13. Kamarajah SK, Bundred JR, Marc OS et al (2020b) A systematic review and network meta-analysis of different surgical approaches for pancreaticoduodenectomy. HPB (Oxford) 22(3):329-339

14. Klompmaker S, van Hilst J, Wellner UF et al (2020) Outcomes after minimally-invasive versus open pancreatoduodenectomy: a Pan-European propensity score matched study. Ann Surg 271(2):356-363

15. Liberati A, Altman DG, Tetzlaff J et al (2009) The PRISMA statement for reporting systematic reviews and meta-analyses of studies that evaluate healthcare interventions: explanation and elaboration. BMJ 21(339):b2700

16. Goossen K, Tenckhoff S, Probst P et al (2019) Optimal literature search for systematic reviews in surgery. Langenbecks Arch Surg 403(1):119-129

17. Wente MN, Bassi C, Dervenis C et al (2007) Delayed gastric emptying (DGE) after pancreatic surgery: a suggested definition by the international study group of pancreatic surgery (ISGPS). Surgery 142:761-768

18. Clavien PA, Barkun J, de Oliveira ML et al (2009) The ClavienDindo classification of surgical complications: five-year experience. Ann Surg 250:187-196
19. Sterne JA, Hernan MA, Reeves BC et al (2016) ROBINS-I: a tool for assessing risk of bias in non-randomised studies of interventions. BMJ 355:4919. https://doi.org/10.1136/bmj.i4919

20. Higgins JP, Altman DG, Gotzsche PC et al (2011) the cochrane collaboration's tool for assessing risk of bias in randomised trials. BMJ 343:d5928

21. Mills EJ, Thorlund K, Ioannidis JP (2013) Demystifying trial networks and network meta-analysis. BMJ 346:f2914

22. Sutton AJ, Abrams KR (2001) Bayesian methods in meta-analysis and evidence synthesis. Stat Methods Med Res 10(4):277-303

23. Aiolfi A, Cavalli M, Micheletto G, Lombardo F, Bonitta G, Morlacchi A, Bruni PG, Campanelli G, Bona D (2019) Primary inguinal hernia: systematic review and Bayesian network meta-analysis comparing open, laparoscopic transabdominal preperitoneal, totally extraperitoneal, and robotic preperitoneal repair. Hernia 23(3):473-484

24. Friede T, Röver C, Wandel S et al (2017) Meta-analysis of few small studies in orphan diseases. Res Synth Methods 8(1):79-91

25. Dias S, Welton NJ, Caldwell DM et al (2010) Checking consistency in mixed treatment comparison meta-analysis. Stat Med 29(7-8):932-944

26. Turner RM, Davey J, Clarke MJ et al (2012) Predicting the extent of heterogeneity in meta-analysis, using empirical data from the cochrane database of systematic reviews. Int J Epidemiol 41(3):818-827

27. Higgins JP, Thompson SG, Deeks JJ et al (2003) Measuring inconsistency in meta-analyses. BMJ 327(7414):557-560

28. Smith BJ (2007) boa: An R package for MCMC output convergence assessment and posterior inference. J Stat Softw 21:1-37. https://doi.org/10.18637/jss.v021.i11

29. R Core Team. R: A Language and Environment for Statistical Computing. R Foundation for Statistical Computing; Vienna, Austria. Available online: https://www.R-project.org/. (Accessed on 30 November 2018)

30. Asbun HJ, Stauffer JA (2012) Laparoscopic vs. open pancreaticoduodenectomy: overall outcomes and severity of complications using the accordion severity grading system. J Am Coll Surg 215(6):810-819

31. Croome KP, Farnell MB, Que FG et al (2014) Total laparoscopic pancreaticoduodenectomy for pancreatic ductal adenocarcinoma: oncologic advantages over open approaches? Ann Surg 260(4):633-638

32. Dokmak S, Ftériche FS, Aussilhou B et al (2015) Laparoscopic pancreaticoduodenectomy should not be routine for resection of periampullary tumors. J Am Coll Surg 220(5):831-838

33. Sharpe SM, Talamonti MS, Wang CE et al (2015) Early national experience with laparoscopic pancreaticoduodenectomy for ductal adenocarcinoma: a comparison of laparoscopic pancreaticoduodenectomy and open pancreaticoduodenectomy from the national cancer data base. J Am Coll Surg 221(1):175-184

34. Speicher PJ, Nussbaum DP, White RR et al (2014) Defining the learning curve for team-based laparoscopic pancreaticoduodenectomy. Ann Surg Oncol 21(12):4014-4019

35. Tee MC, Croome KP, Shubert CR et al (2015) Laparoscopic pancreatoduodenectomy does not completely mitigate increased perioperative risks in elderly patients. HPB (Oxford) 17(10):909-918

36. Tran TB, Dua MM, Worhunsky DJ et al (2016) The first decade of laparoscopic pancreaticoduodenectomy in the United States: costs and outcomes using the nationwide inpatient sample. Surg Endosc 30(5): 1778-1783

37. Senthilnathan P, Chinnusamy P, Ramanujam A et al (2015) Comparison of pathological radicality between open and laparoscopic pancreaticoduodenectomy in a tertiary centre. Indian J Surg Oncol 6(1):20-25 
38. Tan C-L (2015) Outcome and costs of laparoscopic pancreaticoduodenectomy during the initial learning curve vs. laparotomy. WJG 21(17):5311

39. Song KB, Kim SC, Hwang DW et al (2015) Matched case-control analysis comparing laparoscopic and open pylorus-preserving pancreaticoduodenectomy in patients with periampullary tumors. Ann Surg 262(1):146-155

40. Kantor O, Talamonti MS, Sharpe S et al (2017) Laparoscopic pancreaticoduodenectomy for adenocarcinoma provides shortterm oncologic outcomes and long-term overall survival rates similar to those for open pancreaticoduodenectomy. Am J Surg 213(3):512-515

41. Delitto D, Luckhurst CM, Black BS et al (2016) Oncologic and perioperative outcomes following selective application of laparoscopic pancreaticoduodenectomy for periampullary malignancies. J Gastrointest Surg 20(7):1343-1349

42. Stauffer JA, Coppola A, Mody K et al (2016) Laparoscopic versus open distal pancreatectomy for pancreatic adenocarcinoma. World J Surg 40(6):1477-1484

43. Conrad C, Basso V, Passot G et al (2017) Comparable long-term oncologic outcomes of laparoscopic versus open pancreaticoduodenectomy for adenocarcinoma: a propensity score weighting analysis. Surg Endosc 31(10):3970-3978

44. Chopinet S, Fuks D, Rinaudo M et al (2018) Postoperative bleeding after laparoscopic pancreaticoduodenectomy: the achilles' heel? World J Surg 42(4):1138-1146

45. Palanivelu C, Senthilnathan P, Sabnis SC et al (2017) Randomized clinical trial of laparoscopic versus open pancreatoduodenectomy for periampullary tumours: Laparoscopic versus open pancreatoduodenectomy for periampullary tumours. Br J Surg 104(11):1443-1450

46. Chapman BC, Gajdos C, Hosokawa P et al (2018) Comparison of laparoscopic to open pancreaticoduodenectomy in elderly patients with pancreatic adenocarcinoma. Surg Endosc 32(5):2239-2248

47. Poves I, Burdío F, Morató O et al (2018) Comparison of perioperative outcomes between laparoscopic and open approach for pancreatoduodenectomy: the PADULAP randomized controlled trial. Ann Surg 268(5):731-739

48. Chen X-M, Sun D-L, Zhang Y (2018) Laparoscopic versus open pancreaticoduodenectomy combined with uncinated process approach: a comparative study evaluating perioperative outcomes (Retrospective cohort study). Internat J Surg 51:170-173

49. Kuesters S, Chikhladze S, Makowiec F et al (2018) Oncological outcome of laparoscopically assisted pancreatoduodenectomy for ductal adenocarcinoma in a retrospective cohort study. Internat $\mathbf{J}$ Surg 55:162-166

50. Meng L-W, Cai Y-Q, Li Y-B et al (2018) Comparison of laparoscopic and open pancreaticoduodenectomy for the treatment of nonpancreatic periampullary adenocarcinomas: surgical laparoscopy. Endosc Percut Tech 28(1):56-61

51. van Hilst J, de Rooij T, Bosscha K et al (2019) Laparoscopic versus open pancreatoduodenectomy for pancreatic or periampullary tumours (LEOPARD-2): a multicentre, patient-blinded, randomised controlled phase 2/3 trial. Lancet Gastroenterol Hepatol 4(3):199-207

52. Buchs NC, Addeo P, Bianco FM et al (2011) Robotic versus open pancreaticoduodenectomy: a comparative study at a single institution. World J Surg 35(12):2739-2746

53. Lai ECH, Yang GPC, Tang CN (2012) Robot-assisted laparoscopic pancreaticoduodenectomy versus open pancreaticoduodenectomy - a comparative study. Internat J Surg 10(9):475-479

54. Baker EH, Ross SW, Seshadri R et al (2016) Robotic pancreaticoduodenectomy: comparison of complications and cost to the open approach: Robotic whipple cost analysis. Int J Med Robotics Comput Assist Surg 12(3):554-560
55. Chen S, Chen J-Z, Zhan Q et al (2015) Robot-assisted laparoscopic versus open pancreaticoduodenectomy: a prospective, matched, mid-term follow-up study. Surg Endosc 29(12):3698-3711

56. Girgis MD, Zenati MS, Steve J et al (2017) Robotic approach mitigates perioperative morbidity in obese patients following pancreaticoduodenectomy. HPB 19(2):93-98

57. Boggi U, Napoli N, Costa F et al (2016) Robotic-assisted pancreatic resections. World J Surg 40(10):2497-2506

58. McMillan MT, Zureikat AH, Hogg ME et al (2017) a propensity score-matched analysis of robotic vs. open pancreatoduodenectomy on incidence of pancreatic fistula. JAMA Surg 152(4):327

59. Zureikat AH, Postlewait LM, Liu Y et al (2016) A multi-institutional comparison of perioperative outcomes of robotic and open pancreaticoduodenectomy. Ann Surg 264(4):640-649

60. Varley PR, Zenati MS, Klobuka A et al (2019) Does robotic pancreaticoduodenectomy improve outcomes in patients with high risk morphometric features compared to the open approach. HPB 21(6):695-701

61. Kauffmann EF, Napoli N, Menonna F et al (2019) A propensity score-matched analysis of robotic versus open pancreatoduodenectomy for pancreatic cancer based on margin status. Surg Endosc 33(1):234-242

62. Napoli N, Kauffmann EF, Menonna F et al (2018) Robotic versus open pancreatoduodenectomy: a propensity score-matched analysis based on factors predictive of postoperative pancreatic fistula. Surg Endosc 32(3):1234-1247

63. Wang S-E, Shyr B-U, Chen S-C et al (2018) Comparison between robotic and open pancreaticoduodenectomy with modified Blumgart pancreaticojejunostomy: a propensity score-matched study. Surgery 164(6):1162-1167

64. Cai J, Ramanathan R, Zenati MS et al (2020) Robotic pancreaticoduodenectomy is associated with decreased clinically relevant pancreatic fistulas: a propensity-matched analysis. J Gastrointest Surg 24(5):1111-1118

65. Marino MV, Podda M, Gomez Ruiz M et al (2020) Roboticassisted versus open pancreaticoduodenectomy: the results of a case-matched comparison. J Robotic Surg 14(3):493-502

66. Liu R, Zhang T, Zhao Z-M et al (2017) The surgical outcomes of robot-assisted laparoscopic pancreaticoduodenectomy versus laparoscopic pancreaticoduodenectomy for periampullary neoplasms: a comparative study of a single center. Surg Endosc 31(6):2380-2386

67. Nassour I, Wang SC, Porembka MR et al (2017) Robotic versus laparoscopic pancreaticoduodenectomy: a NSQIP analysis. J Gastrointest Surg 21(11):1784-1792

68. Nassour I, Choti MA, Porembka MR et al (2018) Robotic-assisted versus laparoscopic pancreaticoduodenectomy: oncological outcomes. Surg Endosc 32(6):2907-2913

69. Zimmerman AM, Roye DG, Charpentier KP (2018) A comparison of outcomes between open, laparoscopic and robotic pancreaticoduodenectomy. HPB 20(4):364-369

70. Xourafas D, Pawlik TM, Cloyd JM (2018) Independent predictors of increased operative time and hospital length of stay are consistent across different surgical approaches to pancreatoduodenectomy. J Gastrointest Surg 22(11):1911-1919

71. Asbun HJ, Moekotte AL, Vissers FL et al (2020) The miami international evidence-based guidelines on minimally invasive pancreas resection. Ann Surg 271(1):1-14

72. Vollmer CM, Asbun HJ, Barkun J et al (2017) Proceedings of the first international state-of-the-art conference on minimally-invasive pancreatic resection (MIPR). HPB (Oxford) 19(3):171-177

73. Bassi C, Marchegiani G, Dervenis C et al (2017) International Study Group on Pancreatic Surgery (ISGPS). The 2016 update of the International Study Group (ISGPS) definition and grading of postoperative pancreatic fistula: 11 Years After. Surgery 161(3):584-591 
74. Vallance AE, Young AL, Macutkiewicz C et al (2015) Calculating the risk of a pancreatic fistula after a pancreaticoduodenectomy: a systematic review. HPB (Oxford) 17(11):1040-1048

75. Kazanjian KK, Hines OJ, Duffy JP et al (2008) Improved survival following pancreaticoduodenectomy to treat adenocarcinoma of the pancreas: the influence of operative blood loss. Arch Surg 143(12):1166-1171

76. Breukink S, Pierie J, Wiggers T (2006) Laparoscopic versus open total mesorectal excision for rectal cancer. Cochrane Database Syst Rev 18(4):05200

77. Kowalsky SJ, Zenati MS, Steve J et al (2019) A combination of robotic approach and eras pathway optimizes outcomes and cost for pancreatoduodenectomy. Ann Surg 269(6):1138-1145

78. Wang J, Ma R, Churilov L et al (2018) The cost of perioperative complications following pancreaticoduodenectomy: a systematic review. Pancreatology 18(2):208-220
79. Choi M, Hwang HK, Lee WJ et al (2020) Total laparoscopic pancreaticoduodenectomy in patients with periampullary tumors: a learning curve analysis. Surg Endosc. https://doi.org/10.1007/ s00464-020-07684-4 (Online ahead of print)

80. Boone BA, Zenati M, Hogg ME et al (2015) Assessment of quality outcomes for robotic pancreaticoduodenectomy: identification of the learning curve. JAMA Surg 150(5):416-422

81. Hogg ME, Besselink MG, Clavien PA et al (2017) Minimally invasive pancreatic resection organizing committee training in minimally invasive pancreatic resections: a paradigm shift away from "see one, do one, teach one." HPB (Oxford) 19(3):234-245

Publisher's Note Springer Nature remains neutral with regard to jurisdictional claims in published maps and institutional affiliations. 